Habtu Zegeye • David M. Malonza

\title{
Hybrid approximation of solutions of integral equations of the Hammerstein type
}

Received: 18 August 2012 / Accepted: 27 November 2012 / Published online: 18 December 2012

(C) The Author(s) 2012. This article is published with open access at Springerlink.com

Abstract Let $X$ be a uniformly convex and uniformly smooth real Banach space with dual $X^{*}$. Let $F$ : $X \rightarrow X^{*}$ and $K: X^{*} \rightarrow X$ be continuous monotone operators. Suppose that the Hammerstein equation $u+K F u=0$ has a solution in $X$. It is proved that a hybrid-type approximation sequence converges strongly to $u^{*}$, where $u^{*}$ is a solution of the equation $u+K F u=0$. In our theorems, the operator $K$ or $F$ need not be defined on a compact subset of $X$ and no invertibility assumption is imposed on $K$.

Mathematics Subject Classification 47H05 - 47H06 - 47H30 - 47J05 · 47J25

ليكن X فضاء بناخ منتظم التحدّب ومنتظم الملوسة مصحوبأ بفضاءٍ ثنوي

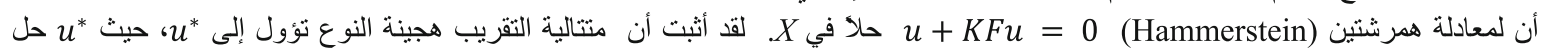

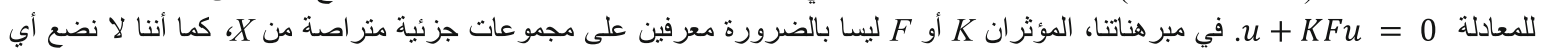

شرط لقابلية $K$ للقلب.

\section{Introduction}

Let $X$ be a real normed linear space with dual $X^{*}$. An operator $A: D(A) \subset X \rightarrow X^{*}$ is said to be $\gamma$-inverse strongly monotone if there exists a positive real number $\gamma$ such that

$$
\langle x-y, A x-A y\rangle \geq \gamma\|A x-A y\|^{2}, \text { for all } x, y \in X .
$$

If $A$ is $\gamma$-inverse strongly monotone, then it is Lipschitz continuous with constant $\frac{1}{\gamma}$, i.e., $\|A x-A y\| \leq$ $\frac{1}{\gamma}\|x-y\|$, for all $x, y \in D(A)$, and it is called uniformly monotone if for each $x, y \in D(A)$ there exists a strictly increasing function $\phi:[0, \infty) \rightarrow[0, \infty)$, with $\phi(0)=0$, such that

$$
\langle x-y, A x-A y\rangle \geq \phi(\|x-y\|) .
$$

H. Zegeye ( $\varangle)$

Department of Mathematics, University of Botswana,

Pvt. Bag 00704, Gaborone, Botswana

E-mail: habtuzh@yahoo.com

D. M. Malonza

Department of Mathematics, Kenyatta University,

43844-001000 Nairobi, Kenya

E-mail: damalo2004@yahoo.co.uk 
An operator $A: D(A) \subset X \rightarrow X^{*}$ is called monotone if for each $x, y \in D(A)$, the following inequality holds:

$$
\langle x-y, A x-A y\rangle \geq 0 .
$$

A close study of monotone operators shows that the class of monotone operators include the class of $\gamma$-inverse strongly monotone and uniformly monotone operators.

A monotone operator $A$ is said to be maximal if its graph $G(A):=\{(x, y): y \in A x\}$ is not properly contained in the graph of any other monotone operator. It is known that a monotone operator $A$ is maximal if and only if for $\left(x, x^{*}\right) \in X \times X^{*},\left\langle x-y, x^{*}-y^{*}\right\rangle \geq 0$ for every $\left(y, y^{*}\right) \in G(A)$ implies $x^{*} \in A(x)$. An operator $A$ from $D(A)=C \subset X$ into $X^{*}$ is said to be hemicontinuous if for all $x, y \in C$, the mapping $f$ of $[0,1]$ into $X^{*}$ defined by $f(t)=A(t x+(1-t) y)$ is continuous with respect to the weak* topology of $X^{*}$. It is well known that a monotone and hemicontinuous operator with $D(A)=X$ is maximal [25].

The mapping $A: X \rightarrow X^{*}$ is said to be angle-bounded with constant $\alpha \geq 0$ if $|\langle y, A x\rangle-\langle x, A y\rangle| \leq$ $2 \alpha\langle x, A x\rangle^{\frac{1}{2}}\langle y, A y\rangle^{\frac{1}{2}}, \forall x, y \in X$. The class of angle-bounded operators is a subclass of that of the monotone operators.

The notion of monotone operators was introduced by Zarantonello [22], Minty [12] and Kacurovskii [9]. Monotonicity conditions in the context of variational methods for nonlinear operator equations were also used by Vainberg and Kacurovskii [20]. The notion has been extended to Banach spaces by several authors.

The generalized formulation of many boundary value problems for ordinary and partial differential equations leads to the operator equations of the type

$$
\langle z, A x\rangle=\langle z, b\rangle, \forall z \in X,
$$

which is equivalent to the equality of functionals on $X$, i.e., the equality of elements of $X^{*}$ :

$$
A x=b,
$$

where $A$ is a monotone-type operator acting from a Banach space $X$ into $X^{*}$. Without loss of generality, we may assume $b=0$. It is known that the solution of the equation $A x=0$ (i.e., $\langle z, A x\rangle=0, \forall z \in X$ ) is the solution of variational inequality $\langle z-x, A x\rangle \geq 0, \forall z \in X$. Therefore, the theory of monotone operators and its applications to nonlinear partial differential equations, evolution equations and variational inequalities are related and have evolved into a substantial topic in nonlinear functional analysis.

One important application of solving (1.4) is finding the zero of the so-called equation of Hammerstein type (see e.g., [6]), where a nonlinear integral equation of Hammerstein type is one of the form:

$$
u(x)+\int_{\Omega} k(x, y) f(y, u(y)) \mathrm{d} y=h(x),
$$

where $\mathrm{d} y$ is a $\sigma$-finite measure on the measure space $\Omega$; the real kernel $k$ is defined on $\Omega \times \Omega, f$ is a real-valued function defined on $\Omega \times \Re$ and is, in general, nonlinear and $h$ is a given function on $\Omega$. If we now define an operator $K$ by

$$
K v(x):=\int_{\Omega} k(x, y) v(y) \mathrm{d} y ; x \in \Omega,
$$

and the so-called superposition or Nemytskii operator by $F u(y):=f(y, u(y))$, then the integral equation (1.5) can be given in the operator theoretic form as follows:

$$
u+K F u=0,
$$

where, without loss of generality, we have taken $h \equiv 0$. The Nemytskii operator $F$ is well defined on a given space $X$ of functions on $\Omega$, and that for each element $u$ of $X, F(u)$ lies in a conjugate space $X^{*}$. If the linear operator $K$ maps the space $X^{*}$ into the space $X$, the composition $K F$ of the two operators is well defined and maps $X$ into itself. Given $h$ in the function space $X$, the integral equation then asks for some $u$ in $X$ such that $(I+K F)(u)=h$. We note that if $K$ and $F$ are monotone, then $A:=I+K F$ need not be necessarily be monotone. 
For the iterative approximation of solutions of Eq. (1.4), the monotonicity of $A$ is crucial. The Mann iteration scheme (see e.g., [11]) and the Ishikawa iteration scheme (see e.g., [7]) have successfully been employed (see e.g., $[3,5,8,15,16,18,26])$. Attempts to apply these methods to Eq. (1.6) have not provided satisfactory results. In particular, the recursion formulas obtained involved $K^{-1}$ (see e.g., [15]) and this is not convenient for applications. Part of the difficulty is the fact that the composition of two monotone operators need not be monotone.

In the special case in which the operators are defined on subsets $D$ of $H$, a Hilbert space, which are compact (or more generally, angle-bounded), Breżis and Browder [2] have proved the strong convergence of a suitably defined Galerkin approximation to a solution of (1.6).

Recently, Chidume and Zegeye [4] have introduced a method which contains an auxiliary operator, defined in an appropriate real Banach space in terms of $K$ and $F$, which, under certain conditions, is uniformly monotone, whenever $K$ and $F$, and whose zeros, are solutions of Eq. (1.6). This leads to the following concern.

Is it possible to construct a convergent iterative sequence which contains an auxiliary operator, defined in an appropriate real Banach space in terms of $K$ and $F$, which, under certain conditions, is monotone, whenever $K$ and $F$, and whose zeros, are solutions of Eq. (1.6)?

It is our purpose in this paper to introduce an auxiliary operator which is monotone, whenever $K$ and $F$ are, and to construct an iterative procedure which converges strongly to a solution of Eq. (1.6). In our theorems, the operator $K$ or $F$ need not be defined on compact or angle-bounded (see e.g., [13]) subset of a uniformly convex and uniformly smooth Banach space $X$, and no invertibility assumption is imposed on $K$.

\section{Preliminaries}

Let $X$ be a normed linear space with $\operatorname{dim} X \geq 2$. The modulus of smoothness of $X$ is the function $\rho_{X}$ : $[0, \infty) \rightarrow[0, \infty)$ defined by

$$
\rho_{X}(\tau):=\sup \left\{\frac{\|x+y\|+\|x-y\|}{2}-1:\|x\|=1 ;\|y\|=\tau\right\} .
$$

The space $X$ is said to be smooth if $\rho_{X}(\tau)>0, \forall \tau>0$ and $X$ is called uniformly smooth if and only if $\lim _{t \rightarrow 0^{+}} \frac{\rho_{X}(t)}{t}=0$.

The modulus of convexity of $X$ is the function $\delta_{X}:(0,2] \rightarrow[0,1]$ defined by

$$
\delta_{X}(\epsilon):=\inf \left\{1-\left|\frac{x+y}{2}\right|:|x|=|y|=1 ; \epsilon=|x-y|\right\} .
$$

$X$ is called uniformly convex if and only if $\delta_{X}(\epsilon)>0$ for every $\epsilon \in(0,2]$.

In the sequel, we shall need the following result:

Lemma 2.1 [23] Let $X$ be a uniformly convex Banach space and $B_{R}(0)$ be a closed ball of $X$. Then, there exists a continuous strictly increasing convex function $g:[0, \infty) \rightarrow[0, \infty)$ with $g(0)=0$ such that

$$
\left\|\alpha_{1} x_{1}+\cdots+\alpha_{N} x_{N}\right\|^{2} \leq \alpha_{1}\left\|x_{1}\right\|^{2}+\cdots+\alpha_{N}\left\|x_{N}\right\|^{2}-\alpha_{i} \alpha_{j} g\left(\left\|x_{i}-x_{j}\right\|\right),
$$

for each $\alpha_{i} \in(0,1)$ and $x_{i} \in B_{R}(0)$, for $i=1,2, \ldots, N$.

Let $X$ be a smooth Banach space. The function $\phi: X \times X \rightarrow \mathbb{R}$ defined by

$$
\phi(x, y)=\|x\|^{2}-2\langle x, J y\rangle+\|y\|^{2} \quad \text { for any } x, y \in X,
$$

was studied by Alber [1], Kamimula and Takahashi [17] and Reich [14], where $J$ is the normalized duality mapping from $E$ to $2^{E^{*}}$ defined by $J x:=\left\{f^{*} \in E^{*}:\left\langle x, f^{*}\right\rangle=\|x\|^{2}=\left\|f^{*}\right\|^{2}\right\}$, in which $\langle.,$.$\rangle denotes the$ duality pairing. It is well known that if $E$ is smooth, then $J$ is single valued and if $E$ has uniformly Gâteaux differentiable norm, then $J$ is uniformly continuous on bounded subsets of $E$. Moreover, if $E$ is a reflexive and strictly convex Banach space with a strictly convex dual, then $J^{-1}$ is single valued, one-to-one, surjective, and is the duality mapping from $E^{*}$ into $E$ and thus $J J^{-1}=I_{E^{*}}$ and $J^{-1} J=I_{E}$ (see [19]).

It is obvious from the definition of the function $\phi$ that

$$
(\|x\|-\|y\|)^{2} \leq \phi(x, y) \leq(\|x\|+\|y\|)^{2} \quad \text { for any } x, y \in X .
$$


We remark that in a Hilbert space $H,(2.1)$ reduces to $\phi(x, y)=\|x-y\|^{2}$, for any $x, y \in H$.

Let $X$ be a reflexive, strictly convex and smooth Banach space and let $C$ be a nonempty, closed and convex subset of $X$. The generalized projection mapping, which was introduced by Alber [1], is the mapping $\Pi_{C}: X \rightarrow C$ that assigns an arbitrary point $x \in X$ to the minimizer, $\bar{x}$, of $\phi(., x)$, that is, $\Pi_{C} x=\bar{x}$, where $\bar{x}$ is the solution to the minimization problem

$$
\phi(\bar{x}, x)=\min \{\phi(y, x), y \in C\} .
$$

Lemma 2.2 [1] Let $C$ be a nonempty, closed and convex subset of a real reflexive, strictly convex, and smooth Banach space $X$ and let $x \in X$. Then there exists a unique element $x_{0} \in C$ such that $\phi\left(x_{0}, x\right)=\min \{\phi(z, x)$ : $z \in C\}$.

Lemma 2.3 [17] Let $X$ be a real smooth and uniformly convex Banach space and let $\left\{x_{n}\right\}$ and $\left\{y_{n}\right\}$ be two sequences of $X$. If either $\left\{x_{n}\right\}$ or $\left\{y_{n}\right\}$ is bounded and $\phi\left(x_{n}, y_{n}\right) \rightarrow 0$ as $n \rightarrow \infty$, then $x_{n}-y_{n} \rightarrow 0$, as $n \rightarrow \infty$.

Lemma 2.4 [1] Let $C$ be a convex subset of a real smooth Banach space $X$. Let $x \in X$. Then $x_{0}=\Pi_{C} x$ if and only if

$$
\left\langle z-x_{0}, J x-J x_{0}\right\rangle \leq 0, \forall z \in C .
$$

We make use of the function $V: X \times X^{*} \rightarrow \mathbb{R}$ defined by

$$
V\left(x, x^{*}\right)=\|x\|^{2}-2\left\langle x, x^{*}\right\rangle+\|x\|^{2}, \quad \text { for all } x \in X \text { and } x^{*} \in X,
$$

studied by Alber [1]. That is, $V\left(x, x^{*}\right)=\phi\left(x, J^{-1} x^{*}\right)$ for all $x \in X$ and $x^{*} \in X^{*}$.

We know the following lemma related to the function $V$.

Lemma 2.5 [1] Let $X$ be reflexive strictly convex and smooth Banach space with $X^{*}$ as its dual. Then

$$
V\left(x, x^{*}\right)+2\left\langle J^{-1} x^{*}-x, y^{*}\right\rangle \leq V\left(x, x^{*}+y^{*}\right),
$$

for all $x \in X$ and $x^{*}, y^{*} \in X^{*}$.

Lemma 2.6 [24] Let $X$ be a smooth, strictly convex and reflexive real Banach space with $X^{*}$ as its dual. Let $A: X \rightarrow X^{*}$ be a continuous monotone mapping. For $r>0$ and $x \in X$, define the operator $F_{r}: X \rightarrow X$ as follows:

$$
F_{r} x:=\left\{z \in X:\langle y-z, A z\rangle+\frac{1}{r}\langle y-z, J z-J x\rangle \geq 0, \forall y \in X\right\},
$$

for all $x \in X$. Then the following hold:

(1) $F_{r}$ is single valued;

(2) $F\left(F_{r}\right)=N(A)$, where $F\left(F_{r}\right):=\left\{z \in X: F_{r}(z)=z\right\}$ and $N(A):=\{z \in X: A z=0\}$;

(3) $\phi\left(p, F_{r} x\right)+\phi\left(F_{r} x, x\right) \leq \phi(p, x)$, for $p \in F\left(F_{r}\right)$;

(4) $N(A)$ is closed and convex.

Lemma 2.7 [21] Let $\left\{a_{n}\right\}$ be a sequence of nonnegative real numbers satisfying the following relation:

$$
a_{n+1} \leq\left(1-\gamma_{n}\right) a_{n}+\sigma_{n}, \quad n \geq 0,
$$

where (i) $\left\{\gamma_{n}\right\} \subset[0,1], \sum \gamma_{n}=\infty$; (ii) $\limsup _{n \rightarrow \infty} \frac{\sigma_{n}}{\gamma_{n}} \leq 0$. Then, $a_{n} \rightarrow 0$ as $n \rightarrow \infty$.

Lemma 2.8 [10] Let $\left\{a_{n}\right\}$ be sequences of real numbers such that there exists a subsequence $\left\{n_{i}\right\}$ of $\{n\}$, and $a_{n_{i}}<a_{n_{i}+1}$ for all $i \in N$. Then there exists a nondecreasing sequence $\left\{m_{k}\right\} \subset N$ such that $m_{k} \rightarrow \infty$ and the following properties are satisfied by all (sufficiently large) numbers $k \in N$ :

$$
a_{m_{k}} \leq a_{m_{k}+1} \text { and } a_{k} \leq a_{m_{k}+1} .
$$

In fact, $m_{k}=\max \left\{j \leq k: a_{j}<a_{j+1}\right\}$. 


\section{Main result}

\subsection{Convergence theorems in Banach spaces}

We first prove the following technical lemma.

Lemma 3.1 Let $X$ be a uniformly smooth and uniformly convex real Banach space with dual $X^{*}$. Let $F: X \rightarrow$ $X^{*}$ and $K: X^{*} \rightarrow X$ be continuous monotone operators. Let $E:=X \times X^{*}$ with norm $\|z\|_{E}^{2}=\|u\|_{X}^{2}+\|v\|_{X^{*}}^{2}$, for $z=(u, v) \in E$ and define an operator $T: E \rightarrow E^{*}$ by

$$
T z:=T(u, v)=(F u-v, u+K v) .
$$

Then $T$ is a continuous monotone operator.

Proof Clearly, continuity of $K$ and $F$ imply that $T$ is continuous. Moreover, for $z_{1}=\left(u_{1}, v_{1}\right) \in E$ and $z_{2}^{*}=\left(u_{2}^{*}, v_{2}^{*}\right) \in E^{*}$, we have that $\left\langle z_{1}, z_{2}^{*}\right\rangle=\left\langle u_{1}, u_{2}^{*}\right\rangle+\left\langle v_{1}, v_{2}^{*}\right\rangle$. Thus using the fact that $K$ and $F$ are monotone, we get the following estimates:

$$
\begin{aligned}
\left\langle T z_{1}-T z_{2}, z_{1}-z_{2}\right\rangle= & \left\langle\left(F u_{1}-v_{1}, u_{1}+K v_{1}\right)-\left(F u_{2}-v_{2}, u_{2}+K v_{2}\right),\right. \\
& \left.\left(u_{1}, v_{1}\right)-\left(u_{2}, v_{2}\right)\right\rangle \\
= & \left\langle F u_{1}-F u_{2}-\left(v_{1}-v_{2}\right), u_{1}-u_{2}\right\rangle \\
& +\left\langle K v_{1}-K v_{2}+\left(u_{1}-u_{2}\right), v_{1}-v_{2}\right\rangle \\
= & \left\langle F u_{1}-F u_{2}, u_{1}-u_{2}\right\rangle-\left\langle v_{1}-v_{2}, u_{1}-u_{2}\right\rangle \\
& +\left\langle K v_{1}-K v_{2}, v_{1}-v_{2}\right\rangle+\left\langle u_{1}-u_{2}, v_{1}-v_{2}\right\rangle \\
\geq & 0,
\end{aligned}
$$

and hence $T$ is monotone, completing the proof of the lemma:

Let $X$ be a uniformly convex and uniformly smooth real Banach space with dual $X^{*}$. Let $F: X \rightarrow X^{*}$ and $K: X^{*} \rightarrow X$ be continuous monotone operators. Let $E$ and $T$ be as in Lemma 3.1. For $r>0$, define:

$$
T_{r} x:=\left\{z \in E:\langle y-z, T z\rangle+\frac{1}{r}\langle y-z, J z-J x\rangle \geq 0, \forall y \in E\right\},
$$

for all $x \in E$. Then in what follows, we shall study the following iteration process.

$$
\left\{\begin{array}{l}
x_{0}=w \in E \text { chosen arbitrarily; } \\
w_{n}=T_{r_{n}} x_{n} \\
x_{n+1}=J^{-1}\left(\alpha_{n} J w+\left(1-\alpha_{n}\right) J w_{n}\right)
\end{array}\right.
$$

where $J$ is the normalized duality mapping on $E ; \alpha_{n} \in(0,1)$ satisfying $\lim _{n \rightarrow \infty} \alpha_{n}=0, \sum_{n=1}^{\infty} \alpha_{n}=\infty$; $\left\{r_{n}\right\} \subset\left[c_{1}, \infty\right)$ for some $c_{1}>0$; for all $n \in \mathbb{N}$.

Theorem 3.2 Let $X$ be a uniformly convex and smooth real Banach space with dual $X^{*}$. Let $F: X \rightarrow X^{*}$ and $K: X^{*} \rightarrow X$ be continuous and bounded monotone operators. Assume that $0=u+K F u$ has $a$ solution in $X$. Let $\left\{x_{n}\right\}_{n \geq 0}$ be a sequence defined by (3.3). Then, the sequence $\left\{x_{n}\right\}_{n \geq 0}$ converges strongly to $x^{*}=\left[u^{*}, v^{*}\right] \in E$, where $u^{*}$ is a solution of the equation $0=u+K F u$ and $v^{*}=\bar{F} u^{*}$.

Proof Observe that $u^{*}$ is the solution of $0=u+K F u$, in $X$ if and only if $x^{*}=\left[u^{*}, v^{*}\right] \in E$ is a solution of $0=T z$ in E, where $v^{*}=F u^{*}$. Thus, we obtain that $N(T)$ ( null space of $\mathrm{T}$ ) $\neq \emptyset$. Moreover, since by Lemma 3.1 we have that $T$ is continuous and monotone, Lemma 2.6 implies that $N(T)$ is closed and convex. 
Now, put $x^{*}:=\Pi_{N(T)} w$, where $\Pi_{N(T)}$ is the generalized projection from $E$ into $N(T)$. From (3.3), the property of $\phi$ and Lemma 2.6 (3) we get that

$$
\begin{aligned}
\phi\left(x^{*}, x_{n+1}\right)= & \phi\left(x^{*}, J^{-1}\left(\alpha_{n} J w+\left(1-\alpha_{n}\right) J w_{n}\right)\right) \\
= & \phi\left(x^{*}, J^{-1}\left(\alpha_{n} J w+\left(1-\alpha_{n}\right) J T_{r_{n}} x_{n}\right)\right) \\
= & \left\|x^{*}\right\|^{2}-2\left\langle x^{*}, \alpha_{n} J w+\left(1-\alpha_{n}\right) J T_{r_{n}} x_{n}\right\rangle \\
& +\left\|\alpha_{n} J w+\left(1-\alpha_{n}\right) J T_{r_{n}} x_{n}\right\|^{2} \\
\leq & \left\|x^{*}\right\|^{2}-2 \alpha_{n}\left\langle x^{*}, J w\right\rangle-2\left(1-\alpha_{n}\right)\left\langle x^{*}, J T_{r_{n}} x_{n}\right\rangle \\
& +\alpha_{n}\|J w\|^{2}+\left(1-\alpha_{n}\right)\left\|J T_{r_{n}} x_{n}\right\|^{2} \\
\leq & \alpha_{n} \phi\left(x^{*}, w\right)+\left(1-\alpha_{n}\right) \phi\left(x^{*}, T_{r_{n}} x_{n}\right) \\
\leq & \alpha_{n} \phi\left(x^{*}, w\right)+\left(1-\alpha_{n}\right) \phi\left(x^{*}, x_{n}\right) .
\end{aligned}
$$

Thus, by induction,

$$
\phi\left(x^{*}, x_{n+1}\right) \leq \max \left\{\phi\left(x^{*}, x_{0}\right), \phi\left(x^{*}, w\right)\right\}, \quad \forall n \geq 1 .
$$

This implies that $\left\{x_{n}\right\}$ and hence $\left\{w_{n}\right\}$ is bounded. Now, since $w_{n}=T_{r_{n}} x_{n}$, using Lemmas 2.5 and 2.6(3) we obtain that

$$
\begin{aligned}
\phi\left(x^{*}, x_{n+1}\right)= & V\left(x^{*}, J x_{n+1}\right) \\
\leq & V\left(x^{*}, J x_{n+1}-\alpha_{n}\left(J w-J x^{*}\right)\right)-2\left\langle x_{n+1}-x^{*},-\alpha_{n}\left(J w-J x^{*}\right)\right\rangle \\
= & \phi\left(x^{*}, J^{-1}\left(\alpha_{n} J x^{*}+\left(1-\alpha_{n}\right) J w_{n}\right)+2 \alpha_{n}\left\langle x_{n+1}-x^{*}, J w-J x^{*}\right\rangle\right. \\
\leq & \alpha_{n} \phi\left(x^{*}, x^{*}\right)+\left(1-\alpha_{n}\right) \phi\left(x^{*}, w_{n}\right)+2 \alpha_{n}\left\langle x_{n+1}-x^{*}, J w-J x^{*}\right\rangle \\
\leq & \left(1-\alpha_{n}\right)\left[\phi\left(x^{*}, x_{n}\right)-\phi\left(w_{n}, x_{n}\right)\right]+2 \alpha_{n}\left\langle x_{n+1}-x^{*}, J w-J x^{*}\right\rangle \\
= & \left(1-\alpha_{n}\right) \phi\left(x^{*}, x_{n}\right)-\left(1-\alpha_{n}\right) \phi\left(w_{n}, x_{n}\right) \\
& +2 \alpha_{n}\left\langle x_{n+1}-x^{*}, J w-J x^{*}\right\rangle \\
\leq & \left(1-\alpha_{n}\right) \phi\left(x^{*}, x_{n}\right)+2 \alpha_{n}\left\langle x_{n+1}-x^{*}, J w-J x^{*}\right\rangle .
\end{aligned}
$$

The rest of the proof is divided into two parts:

Case 1. Suppose that there exists $n_{0} \in N$ such that $\left\{\phi\left(x^{*}, x_{n}\right)\right\}$ is non-increasing. Then, we obtain that $\left\{\phi\left(x^{*}, x_{n}\right)\right\}$ is convergent.

Moreover, from (3.5) and $\alpha_{n} \rightarrow 0$, we get

$$
\begin{aligned}
\phi\left(w_{n}, x_{n}\right) \leq & \left(\phi\left(x^{*}, x_{n}\right)-\phi\left(x^{*}, x_{n+1}\right)\right)+\alpha_{n} \phi\left(x^{*}, x_{n}\right)+\alpha_{n} \phi\left(w_{n}, x_{n}\right) \\
& +2 \alpha_{n}\left\langle x_{n+1}-x^{*}, J w-J x^{*}\right\rangle \rightarrow 0, \quad \text { as } n \rightarrow \infty .
\end{aligned}
$$

Thus, we have that $\phi\left(w_{n}, x_{n}\right) \rightarrow 0$ and hence by Lemma 2.3 we obtain that

$$
x_{n}-w_{n} \rightarrow 0, \quad \text { as } n \rightarrow \infty .
$$

Furthermore, from the property of $\phi$ and the fact that $\alpha_{n} \rightarrow 0$, as $n \rightarrow \infty$, we have that

$$
\begin{aligned}
\phi\left(w_{n}, x_{n+1}\right) & =\phi\left(w_{n}, J^{-1}\left(\alpha_{n} J w+\left(1-\alpha_{n}\right) J w_{n}\right)\right. \\
& \leq \alpha_{n} \phi\left(w_{n}, w\right)+\left(1-\alpha_{n}\right) \phi\left(w_{n}, w_{n}\right) \\
& \leq \alpha_{n} \phi\left(w_{n}, w\right)+\left(1-\alpha_{n}\right) \phi\left(w_{n}, w_{n}\right) \rightarrow 0, \quad \text { as } n \rightarrow \infty,
\end{aligned}
$$

and hence from Lemma 2.3 we get that

$$
w_{n}-x_{n+1} \rightarrow 0, \quad \text { as } n \rightarrow \infty .
$$

Since $\left\{x_{n+1}\right\}$ is bounded and $E$ is reflexive, we choose a subsequence $\left\{x_{n_{k}+1}\right\}$ of $\left\{x_{n+1}\right\}$ such that $x_{n_{k}+1} \rightarrow z$ and $\lim \sup _{n \rightarrow \infty}\left\langle x_{n+1}-x^{*}, J w-J x^{*}\right\rangle=\lim _{k \rightarrow \infty}\left\langle x_{n_{k}+1}-x^{*}, J w-J x^{*}\right\rangle$. Then, from (3.9) we get that $w_{n_{k}} \rightarrow z$. Moreover, from (3.7) and the uniform continuity of $J$, we get that

$$
J w_{n_{k}}-J x_{n_{k}} \rightarrow 0, \quad \text { as } k \rightarrow \infty .
$$


Now, we show that $z \in T^{-1}(0)$. But from the definition of $w_{n}$ we have that

$$
\left\langle y-w_{n}, T w_{n}\right\rangle+\left\langle y-w_{n}, \frac{J w_{n}-J x_{n}}{r_{n}}\right\rangle \geq 0, \quad \forall y \in E,
$$

and hence

$$
\left\langle y-w_{n_{k}}, T w_{n_{k}}\right\rangle+\left\langle y-w_{n_{k}}, \frac{J w_{n_{k}}-J x_{n_{k}}}{r_{n_{k}}}\right\rangle \geq 0, \quad \forall y \in E .
$$

Set $v_{t}=t y+(1-t) z$ for all $t \in(0,1]$ and $y \in E$ fixed. Now, from (3.12) it follows that

$$
\begin{aligned}
\left\langle v_{t}-w_{n_{k}}, T v_{t}\right\rangle & \geq\left\langle v_{t}-w_{n_{k}}, T v_{t}\right\rangle-\left\langle v_{t}-w_{n_{k}}, T w_{n_{k}}\right\rangle-\left\langle v_{t}-w_{n_{k}}, \frac{J w_{n_{k}}-J x_{n_{k}}}{r_{n_{k}}}\right\rangle \\
& =\left\langle v_{t}-w_{n_{k}}, T v_{t}-T w_{n_{k}}\right\rangle-\left\langle v_{t}-w_{n_{k}}, \frac{J w_{n_{k}}-J x_{n_{k}}}{r_{n_{k}}}\right\rangle .
\end{aligned}
$$

From (3.10), we obtain that $\frac{J w_{n_{k}}-J x_{n_{k}}}{r_{n_{k}}} \rightarrow 0$, as $k \rightarrow \infty$. Since $T$ is monotone, we also have that $\left\langle v_{t}-\right.$ $\left.w_{n_{k}}, T v_{t}-T w_{n_{k}}\right\rangle \geq 0$. Thus, it follows that

$$
0 \leq \lim _{k \rightarrow \infty}\left\langle v_{t}-w_{n_{k}}, T v_{t}\right\rangle=\left\langle v_{t}-z, T v_{t}\right\rangle
$$

and hence

$$
\left\langle y-z, T v_{t}\right\rangle \geq 0, \quad \forall y \in E .
$$

If $t \rightarrow 0$, the continuity of $T$ implies that

$$
\langle y-z, T z\rangle \geq 0, \quad \forall y \in E .
$$

Thus, the maximality of $T$ implies that $z \in T^{-1}(0)$. Then, by Lemma 2.4, we immediately obtain that $\limsup _{n \rightarrow \infty}\left\langle x_{n+1}-x^{*}, J w-J x^{*}\right\rangle=\lim _{k \rightarrow \infty}\left\langle x_{n_{k}+1}-x^{*}, J w-J x^{*}\right\rangle=\left\langle z-x^{*}, J w-J x^{*}\right\rangle \leq 0$. It follows from Lemma 2.7 and (3.6) that $\phi\left(x^{*}, x_{n}\right) \rightarrow 0$, as $n \rightarrow \infty$. Consequently, $x_{n} \rightarrow x^{*}$.

Case 2. Suppose that there exists a subsequence $\left\{n_{i}\right\}$ of $\{n\}$ such that

$$
\phi\left(x^{*}, x_{n_{i}}\right)<\phi\left(x^{*}, x_{n_{i}+1}\right),
$$

for all $i \in N$. Then by Lemma 2.8, there exists a nondecreasing sequence $\left\{m_{k}\right\} \subset N$ such that $m_{k} \rightarrow$ $\infty, \phi\left(x^{*}, x_{m_{k}}\right) \leq \phi\left(x^{*}, x_{m_{k}+1}\right)$ and $\phi\left(x^{*}, x_{k}\right) \leq \phi\left(x^{*}, x_{m_{k}+1}\right)$, for all $k \in N$. From (3.5) and $\alpha_{n} \rightarrow 0$, we have

$$
\begin{aligned}
\phi\left(w_{m_{k}}, x_{m_{k}}\right) \leq & \left(\phi\left(x^{*}, x_{m_{k}}\right)-\phi\left(x^{*}, x_{m_{k}+1}\right)\right)+\alpha_{m_{k}} \phi\left(x^{*}, x_{m_{k}}\right)+\alpha_{m_{k}} \phi\left(w_{m_{k}}, x_{m_{k}}\right) \\
& +2 \alpha_{m_{k}}\left\langle x_{m_{k}+1}-x^{*}, J w-J x^{*}\right\rangle \rightarrow 0, \quad \text { as } k \rightarrow \infty .
\end{aligned}
$$

Thus, we get that $\phi\left(w_{m_{k}}, x_{m_{k}}\right) \rightarrow 0$ and hence by Lemma 2.3 we obtain that

$$
x_{m_{k}}-w_{m_{k}} \rightarrow 0, \quad \text { as } k \rightarrow \infty .
$$

Furthermore, from the property of $\phi$ and the fact that $\alpha_{n} \rightarrow 0$, as $n \rightarrow \infty$, we get that

$$
\begin{aligned}
\phi\left(w_{m_{k}}, x_{m_{k}+1}\right) & =\phi\left(w_{m_{k}}, J^{-1}\left(\alpha_{m_{k}} J w+\left(1-\alpha_{m_{k}}\right) J w_{k_{k}}\right)\right. \\
& \leq \alpha_{m_{k}} \phi\left(w_{m_{k}}, w\right)+\left(1-\alpha_{m_{k}}\right) \phi\left(w_{m_{k}}, w_{m_{k}}\right) \\
& \leq \alpha_{m_{k}} \phi\left(w_{m_{k}}, w\right)+\left(1-\alpha_{m_{k}}\right) \phi\left(w_{m_{k}}, w_{m_{k}}\right) \rightarrow 0, \quad \text { as } k \rightarrow \infty,
\end{aligned}
$$

and hence from Lemma 2.3 we have that $w_{m_{k}}-x_{m_{k}+1} \rightarrow 0$. Thus, by the same argument as in Case 1, we get that

$$
\limsup _{k \rightarrow \infty}\left\langle x_{m_{k}+1}-x^{*}, J w-J x^{*}\right\rangle \leq 0 .
$$


Therefore, from (3.6) we have

$$
\phi\left(x^{*}, x_{m_{k}+1}\right) \leq\left(1-\alpha_{m_{k}}\right) \phi\left(x^{*}, x_{m_{k}}\right)+2 \alpha_{m_{k}}\left\langle x_{m_{k}+1}-x^{*}, J w-J x^{*}\right\rangle .
$$

Since $\phi\left(x^{*}, x_{m_{k}}\right) \leq \phi\left(x^{*}, x_{m_{k}+1}\right)$, we have

$$
\begin{aligned}
\alpha_{m_{k}} \phi\left(x^{*}, x_{m_{k}}\right) & \leq \phi\left(x^{*}, x_{m_{k}}\right)-\phi\left(x^{*}, x_{m_{k}+1}\right)+2 \alpha_{m_{k}}\left\langle x_{m_{k}+1}-x^{*}, J w-J x^{*}\right\rangle \\
& \leq 2 \alpha_{m_{k}}\left\langle x_{m_{k}+1}-x^{*}, J w-J x^{*}\right\rangle .
\end{aligned}
$$

In particular, since $\alpha_{m_{k}}>0$, we get

$$
\phi\left(x^{*}, x_{m_{k}}\right) \leq 2\left\langle x_{m_{k}+1}-x^{*}, J w-J x^{*}\right\rangle .
$$

It follows from (3.15) that $\phi\left(x^{*}, x_{m_{k}}\right) \rightarrow 0$ as $k \rightarrow \infty$. This together with (3.16) implies that $\phi\left(x^{*}, x_{m_{k}+1}\right) \rightarrow$ 0 as $k \rightarrow \infty$. But $\phi\left(x^{*}, x_{k}\right) \leq \phi\left(x^{*}, x_{m_{k}+1}\right)$ for all $k \in N$, which implies that $x_{k} \rightarrow x^{*}$ as $k \rightarrow \infty$. Therefore, from both cases, we conclude that $\left\{x_{n}\right\}$ converges strongly to $x^{*}=\left[u^{*}, v^{*}\right] \in E$, where $u^{*}$ is the solution of the equation $u+K F u=0$. The proof is complete.

If in Theorem 3.2, we put that $X=H$, a real Hilbert space, then we get the following corollary.

Corollary 3.3 Let $H$ be a real Hilbert space. Let $F, K: H \rightarrow H$ be continuous and bounded monotone operators. Let $E:=H \times H$ with norm $\|z\|_{E}^{2}=\|u\|_{H}^{2}+\|v\|_{H}^{2}$, for $z=(u, v) \in E$ and let an operator $T: E \rightarrow E$ be defined by $T z:=T(u, v)=(F u-v, u+K v)$. Let the sequence $\left\{x_{n}\right\}$ be generated by:

$$
\left\{\begin{array}{l}
x_{0}=w \in E \text { chosen arbitrarily; } \\
w_{n}=T_{r_{n}} x_{n} \\
x_{n+1}=\alpha_{n} w+\left(1-\alpha_{n}\right) w_{n}
\end{array}\right.
$$

where $T_{r_{n}} x:=\left\{z \in E:\langle y-z, T z\rangle+\frac{1}{r_{n}}\langle y-z, z-x\rangle \geq 0, \forall y \in E\right\}, \alpha_{n} \in(0,1)$ satisfying $\lim _{n \rightarrow \infty} \alpha_{n}=0, \sum_{n=1}^{\infty} \alpha_{n}=\infty$ and $\left\{r_{n}\right\} \subset\left[c_{1}, \infty\right)$ for some $c_{1}>0 ;$ for all $n \in \mathbb{N}$. Then the sequence $\left\{x_{n}\right\}$ converges strongly to $x^{*}=\left[u^{*}, v^{*}\right] \in E$, where $u^{*}$ is a solution of the equation $0=u+K F u$.

\subsection{Convergence theorems in Hilbert spaces}

Theorem 3.4 Let $H$ be a real Hilbert space. Let $F: H \rightarrow H$ and $K: H \rightarrow H$ be continuous and bounded monotone operators. Let $E:=H \times H$ with norm $\|z\|_{E}^{2}=\|u\|_{H}^{2}+\|v\|_{H}^{2}$, for $z=(u, v) \in E$ and let a map $T: E \rightarrow E$ defined by $T z:=T(u, v)=(F u-v, u+K v)$ be $\gamma$-inverse strongly monotone. Let a sequence $\left\{x_{n}\right\}$ be generated by:

$$
\left\{\begin{array}{l}
x_{0}=w \in E \text { chosen arbitrarily } \\
w_{n}=x_{n}-\gamma_{n} T x_{n} \\
x_{n+1}=\alpha_{n} w+\beta_{n} x_{n}+\lambda_{n} w_{n}
\end{array}\right.
$$

where $\alpha_{n}, \beta_{n}, \gamma_{n}, \lambda_{n} \in(0,1)$ satisfy $\alpha_{n}+\beta_{n}+\lambda_{n}=1$ and $\lim _{n \rightarrow \infty} \alpha_{n}=0, \sum_{n=1}^{\infty} \alpha_{n}=\infty ; 0<\beta \leq \beta_{n}, \lambda_{n}$, for all $n \geq 0$ and $0<a_{0} \leq \gamma_{n} \leq \gamma$, for some $a_{0}, \beta \in \mathbb{R}$. Then the sequence $\left\{x_{n}\right\}$ converges strongly to $x^{*}=\left[u^{*}, v^{*}\right] \in E$, where $u^{*}$ is a solution of the equation $0=u+K F u$ and $v^{*}=F u^{*}$.

Proof Following the argument of proof of Theorem 3.2, we have that $N(T) \neq \emptyset$ which is closed and convex. Now, take $x^{*}:=\Pi_{N(T)} w$, where $\Pi_{N(T)}$ is the generalized projection from $E$ into $N(T)$. From (3.19), Lemma 2.5 and $\gamma$-inverse strong monotonicity of $T$, we get that

$$
\begin{aligned}
\phi\left(x^{*}, w_{n}\right)= & \phi\left(x^{*}, x_{n}-\gamma_{n} T x_{n}\right)=V\left(x^{*}, x_{n}-\gamma_{n} T x_{n}\right) \\
\leq & V\left(x^{*}, x_{n}\right)-2 \gamma_{n}\left\langle\left(x_{n}-\gamma_{n} T x_{n}\right)-x_{n}, T x_{n}\right\rangle \\
& -2 \gamma_{n}\left\langle x_{n}-x^{*}, T x_{n}\right\rangle \\
= & \phi\left(x^{*}, x_{n}\right)-2 \gamma_{n}\left\langle x_{n}-x^{*}, T x_{n}-T x^{*}\right\rangle+2 \gamma_{n}\left\langle T x_{n}-T x^{*}, T x_{n}-T x^{*}\right\rangle \\
\leq & \phi\left(x^{*}, x_{n}\right)-2 \gamma_{n} \gamma\left\|T x_{n}-T x^{*}\right\|^{2}+2 \gamma_{n}^{2}\left\|T x_{n}-x_{n}\right\|^{2} \\
= & \phi\left(x^{*}, x_{n}\right)+2 \gamma_{n}\left(\gamma_{n}-\gamma\right)\left\|T x_{n}-T x^{*}\right\|^{2} \leq \phi\left(x^{*}, x_{n}\right) .
\end{aligned}
$$


Furthermore, from (3.19), the property of $\phi$, Lemma 2.1 and (3.20), we get that

$$
\begin{aligned}
\phi\left(x^{*}, x_{n+1}\right)= & \left.\phi\left(x^{*}, \alpha_{n} w+\beta_{n} x_{n}+\lambda_{n} w_{n}\right)\right) \\
= & \left\|x^{*}\right\|^{2}-2\left\langle x^{*}, \alpha_{n} w+\beta_{n} x_{n}+\lambda_{n} w_{n}\right\rangle \\
& +\left\|\alpha_{n} w+\beta_{n} x_{n}+\lambda_{n} w_{n}\right\|^{2} \\
\leq & \left\|x^{*}\right\|^{2}-2 \alpha_{n}\left\langle x^{*}, w\right\rangle-2 \beta_{n}\left\langle x^{*}, x_{n}\right\rangle-2 \lambda_{n}\left\langle x^{*}, w_{n}\right\rangle \\
& +\alpha_{n}\|w\|^{2}+\beta_{n}\left\|x_{n}\right\|^{2}+\lambda_{n}\left\|w_{n}\right\|^{2}-\beta_{n} \lambda_{n} g\left(\left\|x_{n}-w_{n}\right\|\right) \\
= & \alpha_{n} \phi\left(x^{*}, w\right)+\beta_{n} \phi\left(x^{*}, x_{n}\right)+\lambda_{n} \phi\left(x^{*}, w_{n}\right)-\beta_{n} \lambda_{n} g\left(\left\|x_{n}-w_{n}\right\|\right) \\
\leq & \alpha_{n} \phi\left(x^{*}, w\right)+\beta_{n} \phi\left(x^{*}, x_{n}\right)+\lambda_{n} \phi\left(x^{*}, x_{n}\right)-\beta_{n} \lambda_{n} g\left(\left\|x_{n}-w_{n}\right\|\right) \\
= & \alpha_{n} \phi\left(x^{*}, w\right)+\left(1-\alpha_{n}\right) \phi\left(x^{*}, x_{n}\right)-\beta_{n} \lambda_{n} g\left(\left\|x_{n}-w_{n}\right\|\right) \\
\leq & \alpha_{n} \phi\left(x^{*}, w\right)+\left(1-\alpha_{n}\right) \phi\left(x^{*}, x_{n}\right),
\end{aligned}
$$

Thus, by induction,

$$
\phi\left(x^{*}, x_{n+1}\right) \leq \max \left\{\phi\left(x^{*}, w\right), \phi\left(x^{*}, x_{n}\right)\right\}, \quad \forall n \geq 1 .
$$

This implies that $\left\{x_{n}\right\}$ and hence $\left\{w_{n}\right\}$ is bounded. Now, from (3.19), Lemma 2.5 and (3.20), we obtain that

$$
\begin{aligned}
\phi\left(x^{*}, x_{n+1}\right) & =V\left(x^{*}, x_{n+1}\right) \\
& \leq V\left(x^{*}, x_{n+1}-\alpha_{n}\left(w-x^{*}\right)\right)-2\left\langle x_{n+1}-x^{*},-\alpha_{n}\left(w-x^{*}\right)\right\rangle \\
& =\phi\left(x^{*}, \alpha_{n} x^{*}+\beta_{n} x_{n}+\lambda_{n} w_{n}\right)+2 \alpha_{n}\left\langle x_{n+1}-x^{*}, w-x^{*}\right\rangle \\
& \leq \alpha_{n} \phi\left(x^{*}, x^{*}\right)+\beta_{n} \phi\left(x^{*}, x_{n}\right)+\lambda_{n} \phi\left(x^{*}, w_{n}\right)+2 \alpha_{n}\left\langle x_{n+1}-x^{*}, w-x^{*}\right\rangle \\
& =\beta_{n} \phi\left(x^{*}, x_{n}\right)+\lambda_{n} \phi\left(x^{*}, w_{n}\right)+2 \alpha_{n}\left\langle x_{n+1}-x^{*}, w-x^{*}\right\rangle \\
& \leq \beta_{n} \phi\left(x^{*}, x_{n}\right)+\lambda_{n} \phi\left(x^{*}, x_{n}\right)+2 \alpha_{n}\left\langle x_{n+1}-x^{*}, w-x^{*}\right\rangle \\
& \leq\left(1-\alpha_{n}\right) \phi\left(x^{*}, x_{n}\right)+2 \alpha_{n}\left\langle x_{n+1}-x^{*}, w-x^{*}\right\rangle .
\end{aligned}
$$

The rest of the proof is divided into two parts:

Case 1. Suppose that there exists $n_{0} \in N$ such that $\left\{\phi\left(x^{*}, x_{n}\right)\right\}$ is non-increasing. Then, we obtain that $\left\{\phi\left(x^{*}, x_{n}\right)\right\}$ is convergent. Now, from (3.21) we get that

$$
\beta_{n} \lambda_{n} g\left(\left\|x_{n}-w_{n}\right\|\right) \leq \alpha_{n} \phi\left(x^{*}, w\right)+\left(1-\alpha_{n}\right) \phi\left(x^{*}, x_{n}\right)-\phi\left(x^{*}, x_{n+1}\right) .
$$

Then, using the fact that $\beta_{n}, \lambda_{n}>\beta>0$ for all $n \geq 1$ and $\alpha_{n} \rightarrow 0$, we obtain that $g\left(\left\|x_{n}-w_{n}\right\|\right) \rightarrow 0$ as $n \rightarrow \infty$.

Furthermore, Inequality (3.20) implies that

$$
\begin{aligned}
2 \gamma_{n}\left(\gamma-\gamma_{n}\left\|T x_{n}-T x^{*}\right\|^{2}\right. & \leq \phi\left(x^{*}, x_{n}\right)-\phi\left(x^{*}, w_{n}\right) \\
& =\left\|x_{n}\right\|^{2}-\left\|w_{n}\right\|^{2}-2\left\langle x^{*}, x_{n}-w_{n}\right\rangle \\
& \leq\left\|x_{n}-w_{n}\right\|\left(\left\|x_{n}\right\|+\left\|w_{n}\right\|\right)+2\left\|x^{*}\right\|\left\|x_{n}-w_{n}\right\| .
\end{aligned}
$$

Thus, since $\left\|x_{n}-w_{n}\right\| \rightarrow 0, \quad$ as $n \rightarrow \infty$, we obtain that

$$
\left\|T x_{n}-T x^{*}\right\| \rightarrow 0 \quad \text { as } k \rightarrow \infty
$$

This implies that $T x_{n} \rightarrow T x^{*}=0$.

Moreover, from (3.19) and the fact that $\alpha_{n} \rightarrow 0$ and $x_{n}-w_{n} \rightarrow 0$, we get that

$$
x_{n+1}-x_{n}=\alpha_{n}\left(w-x_{n}\right)+\lambda_{n}\left(w_{n}-x_{n}\right) \rightarrow 0, \quad \text { as } n \rightarrow \infty .
$$

Thus, since $\left\{x_{n+1}\right\}$ is bounded and $E$ is reflexive, we may choose a subsequence $\left\{x_{n_{k}+1}\right\}$ of $\left\{x_{n+1}\right\}$ such that $x_{n_{k}+1} \rightarrow z$ and $\limsup _{n \rightarrow \infty}\left\langle x_{n+1}-x^{*}, w-x^{*}\right\rangle=\lim _{k \rightarrow \infty}\left\langle x_{n_{k}+1}-x^{*}, w-x^{*}\right\rangle$. Then from (3.24), 
we get that $x_{n_{k}} \rightarrow z$. Moreover, for $x \in E$, monotonicity of $T$ implies that $\left\langle x-x_{n_{k}}, T x-T x_{n_{k}}\right\rangle \geq 0$ for all $k \geq 1$, and hence, since

$$
\begin{aligned}
\left|\left\langle x-x_{n_{k}}, T x-T x_{n_{k}}\right\rangle-\left\langle x_{n_{k}}-z, T x\right\rangle\right| \leq & \left|\left\langle x-x_{n_{k}}-(x-z), T x\right\rangle\right| \\
& +\left|\left\langle x_{n_{k}}-z, T x_{n_{k}}\right\rangle\right| \\
\leq & \left|\left\langle x-x_{n_{k}}-(x-z), T x\right\rangle\right| \\
& +\|x-z\|\left\|T x_{n_{k}}\right\| \rightarrow 0, \quad \text { as } k \rightarrow \infty,
\end{aligned}
$$

we obtain that $\langle x-z, T x\rangle \geq 0$. Thus, maximality of $T$ implies that $z \in N(T)$. Therefore, by Lemma 2.4, we immediately obtain that $\lim _{\sup } \operatorname{sum}_{n \rightarrow \infty}\left\langle x_{n+1}-x^{*}, w-x^{*}\right\rangle=\lim _{k \rightarrow \infty}\left\langle x_{n_{k}+1}-x^{*}, w-x^{*}\right\rangle=\left\langle z-x^{*}, J w-\right.$ $\left.J x^{*}\right\rangle \leq 0$, and hence

$$
\limsup _{n \rightarrow \infty}\left\langle x_{n+1}-x^{*}, w-x^{*}\right\rangle \leq 0
$$

Thus, from (3.22) and Lemma 2.7, we obtain that $\phi\left(x^{*}, x_{n}\right) \rightarrow 0$ and hence by Lemma 2.3 we have that $x_{n} \rightarrow 0$ as $n \rightarrow \infty$.

Case 2. Suppose that there exists a subsequence $\left\{n_{i}\right\}$ of $\{n\}$ such that

$$
\phi\left(x^{*}, x_{n_{i}}\right)<\phi\left(x^{*}, x_{n_{i}+1}\right)
$$

for all $i \in N$. Then by Lemma 2.8, there exists a nondecreasing sequence $\left\{m_{k}\right\} \subset N$ such that $m_{k} \rightarrow \infty$, $\phi\left(x^{*}, x_{m_{k}}\right) \leq \phi\left(x^{*}, x_{m_{k}+1}\right)$ and $\phi\left(x^{*}, x_{k}\right) \leq \phi\left(x^{*}, x_{m_{k}+1}\right)$ for all $k \in N$. Now, from (3.21) we get that

$$
\beta_{m_{k}} \lambda_{m_{k}} g\left(\left\|x_{m_{k}}-w_{m_{k}}\right\|\right) \leq \alpha_{m_{k}} \phi\left(x^{*}, w\right)+\left(1-\alpha_{m_{k}}\right) \phi\left(x^{*}, x_{m_{k}}\right)-\phi\left(x^{*}, x_{m_{k}+1}\right) .
$$

Then, using the fact that $\beta_{n}, \lambda_{n}>\beta>0$ and $\alpha_{n} \rightarrow 0$, we obtain that $g\left(\left\|x_{m_{k}}-w_{m_{k}}\right\|\right) \rightarrow 0$ as $k \rightarrow \infty$. Thus, following the method of proof of case 1 , we get that $x_{m_{k}}-w_{m_{k}} \rightarrow 0, T x_{m_{k}} \rightarrow T x^{*}=0$ and $x_{m_{k}}-x_{m_{k}+1} \rightarrow 0$, as $k \rightarrow \infty$, and hence

$$
\limsup _{k \rightarrow \infty}\left\langle x_{m_{k}+1}-x^{*}, w-x^{*}\right\rangle \leq 0 .
$$

Therefore, following the agreement of case 2 of Theorem 3.2 we obtain that $\left\{x_{n}\right\}$ converges strongly to $x^{*}$ and the proof is complete.

Remark 3.5 The method of proof of Theorem 3.4 provides the following explicit algorithm for computing the solution of the equation $0=u+K F u$ in real Hilbert spaces. Take $x_{n}:=\left(z_{n}, y_{n}\right)$, then with initial values $z_{0}, y_{0} \in H$, Scheme (3.19), we get sequences $z_{n}$ and $y_{n}$ given by:

$$
\left\{\begin{array}{l}
z_{0}, y_{0} \in H, \text { chosen arbitrarily; } \\
z_{n+1}=\alpha_{n} z_{0}+\beta_{n} z_{n}+\lambda_{n} z_{n}-\lambda_{n} \gamma_{n}\left(F z_{n}-y_{n}\right) \\
y_{n+1}=\alpha_{n} y_{0}+\beta_{n} y_{n}+\lambda_{n} y_{n}-\lambda_{n} \gamma_{n}\left(z_{n}+K y_{n}\right)
\end{array}\right.
$$

where $\alpha_{n}, \beta_{n}, \gamma_{n}, \lambda_{n} \in(0,1)$ satisfy $\alpha_{n}+\beta_{n}+\lambda_{n}=1$ and $\lim _{n \rightarrow \infty} \alpha_{n}=0, \sum_{n=1}^{\infty} \alpha_{n}=\infty ; 0<\beta \leq$ $\beta_{n}, \lambda_{n}$, for all $n \geq 0$ and $0<a_{0} \leq \gamma_{n} \leq \gamma$, for some $a_{0}, \beta \in \mathbb{R}$. Then the sequence $\left\{x_{n}\right\}$ converges strongly to $x^{*}=\left[u^{*}, v^{*}\right] \in H \times H$, where $u^{*}$ is a solution of the equation $0=u+K F u$ and $v^{*}=F u^{*}$.

The following example gives a prototype of operators $F, K$ and $T$ satisfying conditions of Theorem 3.4.

Example 3.6 Let $F, K:(\mathbb{R},||.) \rightarrow(\mathbb{R},||$.$) be defined by F(x)=2 x$ for some real constant $c$ and $K(x)=$ $3 x+c$. Clearly, $F$ and $K$ are continuous monotone operators. Define $T:\left(\mathbb{R} \times \mathbb{R},\left.\|\cdot\|\right|_{2}\right) \rightarrow\left(\mathbb{R} \times \mathbb{R},\left.\|\cdot\|\right|_{2}\right)$ by 
$T(x, y):=(F x-y, x+K y)=(2 x-y, x+3 y+c)$. Then $T\left(x_{1}, x_{2}\right)=\left(2 x_{1}-x_{2}, x_{1}+3 x_{2}+c\right), T\left(y_{1}, y_{2}\right)=$ $\left(2 y_{1}-y_{2}, y_{1}+3 y_{2}+c\right)$ and $T\left(x_{1}, x_{2}\right)-T\left(y_{1}, y_{2}\right)=\left(2\left(x_{1}-y_{1}\right)-\left(x_{2}-y_{2}\right),\left(x_{1}-y_{1}\right)+3\left(x_{2}-y_{2}\right)\right)$. This implies that

$$
\begin{aligned}
\left\|T\left(x_{1}, x_{2}\right)-T\left(y_{1}, y_{2}\right)\right\|_{2}^{2}= & {\left[2\left(x_{1}-y_{1}\right)-\left(x_{2}-y_{2}\right)\right]^{2}+\left[\left(x_{1}-y_{1}\right)+3\left(x_{2}-y_{2}\right)\right]^{2} } \\
= & 4\left(x_{1}-y_{1}\right)^{2}-4\left(x_{1}-y_{1}\right)\left(x_{2}-y_{2}\right)+\left(x_{2}-y_{2}\right)^{2} \\
& +\left(x_{1}-y_{1}\right)^{2}+6\left(x_{1}-y_{1}\right)\left(x_{2}-y_{2}\right)+9\left(x_{2}-y_{2}\right)^{2} \\
= & 5\left(x_{1}-y_{1}\right)^{2}+2\left(x_{1}-y_{1}\right)\left(x_{2}-y_{2}\right)+9\left(x_{2}-y_{2}\right)^{2} .
\end{aligned}
$$

Case 1. If $\left(x_{1}-y_{1}\right)\left(x_{2}-y_{2}\right) \leq 0$, then we have that

$$
\left\|T\left(x_{1}, x_{2}\right)-T\left(y_{1}, y_{2}\right)\right\|_{2}^{2} \leq 5\left(x_{1}-y_{1}\right)^{2}+9\left(x_{2}-y_{2}\right)^{2} .
$$

Case 2. If $\left(x_{1}-y_{1}\right)\left(x_{2}-y_{2}\right)>0$ and $\left(x_{1}-y_{1}\right) \leq\left(x_{2}-y_{2}\right)$, we have

$$
\begin{aligned}
\left\|T\left(x_{1}, x_{2}\right)-T\left(y_{1}, y_{2}\right)\right\|_{2}^{2} & \leq 5\left(x_{1}-y_{1}\right)^{2}+9\left(x_{2}-y_{2}\right)^{2}+2\left(x_{2}-y_{2}\right)^{2} \\
& \leq 5\left(x_{1}-y_{1}\right)^{2}+11\left(x_{2}-y_{2}\right)^{2},
\end{aligned}
$$

Case 3. If $\left(x_{1}-y_{1}\right)\left(x_{2}-y_{2}\right)>0$ and $\left(x_{1}-y_{1}\right)>\left(x_{2}-y_{2}\right)$, we have

$$
\begin{aligned}
\left\|T\left(x_{1}, x_{2}\right)-T\left(y_{1}, y_{2}\right)\right\|_{2}^{2} & \leq 5\left(x_{1}-y_{1}\right)^{2}+9\left(x_{2}-y_{2}\right)^{2}+2\left(x_{1}-y_{1}\right)^{2} \\
& \leq 7\left(x_{1}-y_{1}\right)^{2}+9\left(x_{2}-y_{2}\right)^{2} .
\end{aligned}
$$

Thus, from Case 1, 2, 3 and (3.27), we obtain that

$$
\left\|T\left(x_{1}, x_{2}\right)-T\left(y_{1}, y_{2}\right)\right\|_{2}^{2} \leq 7\left(x_{1}-y_{1}\right)^{2}+11\left(x_{2}-y_{2}\right)^{2} .
$$

On the other hand, we have that

$$
\begin{aligned}
\left\langle\left(x_{1}, x_{2}\right)-\left(y_{1}, y_{2}\right), T\left(x_{1}, x_{2}\right)-T\left(y_{1}, y_{2}\right)\right\rangle= & \left\langle\left(x_{1}-y_{1}, x_{2}-y_{2}\right),\left(2\left(x_{1}-y_{1}\right)-\left(x_{2}-y_{2}\right),\left(x_{1}-y_{1}\right)+3\left(x_{2}-y_{2}\right)\right\rangle\right. \\
= & \left(x_{1}-y_{1}\right)\left[2\left(x_{1}-y_{1}\right)-\left(x_{2}-y_{2}\right)\right] \\
& +\left(x_{2}-y_{2}\right)\left[\left(x_{1}-y_{1}\right)+3\left(x_{2}-y_{2}\right)\right] \\
= & 2\left(x_{1}-y_{1}\right)^{2}+3\left(x_{2}-y_{2}\right)^{2} \\
\geq & \frac{3}{11}\left[\frac{22}{3}\left(x_{1}-y_{1}\right)^{2}+11\left(x_{2}-y_{2}\right)^{2}\right] \\
\geq & \frac{3}{11}\left[7\left(x_{1}-y_{1}\right)^{2}+11\left(x_{2}-y_{2}\right)^{2}\right]
\end{aligned}
$$

Therefore, from (3.28) and (3.29), we obtain that

$$
\left\langle\left(x_{1}, x_{2}\right)-\left(y_{1}, y_{2}\right), T\left(x_{1}, x_{2}\right)-T\left(y_{1}, y_{2}\right)\right\rangle \geq \frac{3}{11}\left\|T\left(x_{1}, x_{2}\right)-T\left(y_{1}, y_{2}\right)\right\|_{2}^{2} .
$$

Hence, $T$ is $\gamma$-inverse strongly monotone with $\gamma \in\left(0, \frac{3}{11}\right]$.

Open Access This article is distributed under the terms of the Creative Commons Attribution License which permits any use, distribution, and reproduction in any medium, provided the original author(s) and the source are credited. 


\section{References}

1. Alber, Y.: Metric and generalized projection operators in Banach spaces: properties and applications. In: Kartsatos, A.G. (ed.) Theory and Applications of Nonlinear Operators of Accretive and Monotone Type. Leture Notes in Pure and Applied Mathematics, vol. 178, pp. 15-50. Dekker, New York (1996)

2. Breżis, H.; Browder, F.E.: Nonlinear integral equations and systems of Hammerstein type. Bull. Am. Math. Soc. 82, 115-147 (1976)

3. Chang, S.S.; Cho, Y.J.; Zhou, Y.Y.: Iterative sequences with mixed errors for asymptotically quasi-nonexpansive type mappings in Banach spaces. Acta Math. Hung. 100, 147-155 (2003)

4. Chidume, C.E.; Zegeye, H.: Iterative approximation of solutions of nonlinear equations of Hammerstein type. Abstract Appl. Anal. 6, 353-365 (2003)

5. Dominquez Benavides, T.; Lopez Acedo, G.; Xu, H.K.: Iterative solutions for zeros of accretive operators. Math. Nachr. 248/249, 62-71 (2003)

6. Hammerstein, A.: Nichtlineare integralgleichungen nebst anwendungen. Acta Math. Soc. 54, 117-176 (1930)

7. Ishikawa, S.: Fixed points by a new iteration method. Proc. Am. Math. Soc. 44, 147-150 (1974)

8. Jung, J.S.; Morales, C.H.: The Mann process for perturbed $m$-accretive operators in Banach spaces. Nonlinear Anal. 46(2), 231-243 (2001)

9. Kacurovski, R.I.: On monotone operators and convex functionals. Uspekhi Mat. Nauk. 15, 213-215 (1960)

10. Mainge, P.E.: Strong convergence of projected subgradient methods for nonsmooth and non- strictly convex minimization. Set Valued Anal. 16, 899-912 (2008)

11. Mann, W.R.: Mean value methods in iteration. Proc. Am. Math. Soc. 4, 506-510 (1953)

12. Minty, G.J.: Monotone operators in Hilbert spaces. Duke Math. J. 29, 341-346 (1962)

13. Sburlan, P.D.: Nonlinear mappings of monotone type, Editura Academiae, Bucaresti (1978)

14. Reich, S.: A weak convergence theorem for the alternating method with Bergman distance. In: Kartsatos, A.G. (ed.) Theory and Applications of Nonlinear Operators of Accretive and Monotone Type. Leture Notes in Pure and Applied Mathematics, vol. 178, pp. 313-318. Dekker, New York (1996)

15. Reich, S.: Constructive techniques for accretive and monotone operators. In: Applied Nonlinear Analysis, pp. 335-345. Academic Press, New York (1979)

16. Rhoades, B.E.; Soltuz, S.M.: The equivalence between the convergence of Ishikawa and Mann iterations for an asymptotically pseudocontractive map. J. Math. Anal. Appl. 283, 681-688 (2003)

17. Kamimura, S.; Takahashi, W.: Strong convergence of proximal-type algorithm in a Banach space. SIAM J. Optim. 13, 938-945 (2002)

18. Ofoedu, E.U.; Malonza, D.M.: Hybride approximation of nonlinear operator equations and applications to equation of Hammerstein. Appl. Math. Comput. 217, 6019-6030 (2010)

19. Takahashi, W.: Nonlinear Functional Analysis (Japanese). Kindikagaku, Tokyo (1988)

20. Vainberg, M.M.; Kacurovskii, R.I.: On the variational theory of nonlinear operators and equations. Dokl. Akad. Nauk. 129, 1199-1202 (1959)

21. Xu, H.K.: Iterative algorithms for nonlinear operators. J. Lond. Math. Soc. 66, 240-256 (2002)

22. Zarantonello, E.H.: Solving functional equations by contractive averaging. Mathematics Research Center Report \#160, Mathematics Research Center, University of Wisconsin, Madison (1960)

23. Zegeye, H.; Ofoedu, E.U.; Shahzad, N.: Convergence theorems for equilibrium problem, variotional inequality problem and countably infinite relatively quasi-nonexpansive mappings. Appl. Math. Comput. 216, 3439-3449 (2010)

24. Zegeye, H.; Shahzad, N.: Approximating common solution of variational inequality problems for two monotone mappings in Banach spaces. Optim. Lett. doi:10.1007/s11590-010-0235-5

25. Zeidler, E.: Nonlinear Functional Analysis its Applications II/B. Springer, New York (1990)

26. Zhou, H.Y.; Chang, S.S.; Agarwal, R.P.; Cho, Y.J.: Stability results for the Ishikawa iteration procedures. Dyn. Contin. Discret. Impuls. Syst. Ser. A Math. Anal. 9, 477-486 (2002) 